\title{
Conversion of steel by polyphenolic model molecules: Corrosion inhibition mechanism by rutin, esculin, esculetol
}

\author{
Delphine Veys-Renaux ${ }^{\mathrm{a}, *}$, Solenn Reguer ${ }^{\mathrm{b}}$, Ludovic Bellot-Gurlet ${ }^{\mathrm{c}}$, François Mirambet $^{\mathrm{d}}$, \\ Emmanuel Rocca ${ }^{\mathrm{a}}$ \\ ${ }^{a}$ Institut Jean Lamour, UMR 7198, Université de Lorraine-CNRS, BP 70239, Vandoeuvre-lès-Nancy, 54506, France \\ ${ }^{\mathrm{b}}$ DiffAbs Beamline, Synchrotron SOLEIL, L'Orme des Merisiers, Saint-Aubin, BP 48, Gif-sur-Yvette CEDEX, 91192, France \\ ' Sorbonne Universités, MONARIS 'de la Molécule aux Nano-objets: Réactivité, Interactions et Spectroscopies', UMR8233 UPMC-CNRS, Université Pierre et Marie Curie \\ Paris 6, 4 Place Jussieu, Paris, 75252, France \\ ${ }^{\mathrm{d}}$ Centre de Recherche et de Restauration des Musées de France (C2RMF), Palais du Louvre, Porte des Lions, 14 Quai François Mitterrand, Paris, 75001 , France
}

\section{A R T I C L E I N F O}

\section{Keywords:}

Steel

Passive films

Polarization

AFS (EXAFS and XANES)

Raman spectroscopy

\begin{abstract}
A B S T R A C T
This paper focuses on the potential corrosion protection of steel in presence of three polyphenolic model molecules (rutin, esculin, esculetol) in a weak acidic medium $(\mathrm{pH}=4)$. From an electrochemical point of view, the cathodic inhibition was attributed to the oxygen scavenger role of the considered antioxidant compounds. SEM observations, Raman spectroscopy and Synchrotron X-ray absorption spectroscopy revealed furthermore the formation of a surface film by precipitation of iron polyphenolate conversion products, in which iron III is likely coordinated with free-catechol groups. Glycosylated catechol groups are rather adsorbed on a FeOOH layer.
\end{abstract}

\section{Introduction}

Polyphenolic molecules like flavonoids or coumarin derivative are compounds characterized by diverse structures and various biological and chemical activities. In nature, especially in plants, they are frequently found as glycosides, in order to ensure their water solubility and favour their absorption by the organisms. They can have diverse functional roles, including antibiotic and antifouling actions, protection against solar radiation or antioxidant activity $[1,2]$.

Regarding their molecular structure, polyphenolic compounds are characterized by at least one aromatic ring cycle, like in the case of coumarins. Regarding flavonoids family, the two aromatic cycles are linked by a heterocycle containing oxygen atoms. In both classes of molecules, the $\mathrm{OH}$ groups can form an isolated phenol function or a catechol function, also named 1,2-dihydroxybenzene. This catechol group is known to be responsible for the chelating properties of these molecules on many metals [3,4], and metal-catecholate complexes are also present in biological systems, like the catechol dioxygenases $[5,6]$ and metal scavengers or siderophores complexes [7,8].

In the field of metal corrosion inhibition, the use of such natural substances with good ligand properties is a hot research topic. Actually, in the present ecological context, vegetal extracts appear as an alternative to fulfil the conditions of REACH regulation and European directives on the waste water reject. In the literature, various plant extracts [9-11], leave extracts [12], fruit peel extracts [13,14] have been tested to inhibit the oxidation of steel. Furthermore, the first commercialized natural corrosion inhibitors were tannins, a class of natural, non-toxic and biodegradable polyphenolic polymers. However, due to the large number and the variety of molecules contained in natural extracts, the understanding of the mechanisms involved in the corrosion inhibition is tricky. Few papers [15-17] relate the individual effect of the molecules contained in natural extracts. In very acidic conditions, the model of Langmuir-type adsorption of monolayers of molecules on metal is proposed to explain the inhibition of oxidation of metals. Nevertheless, the formation or precipitation of a tridimensional conversion films can sometimes be observed on metals.

The aim of the present paper is to compare the effects on steel (morphological, structural and electrochemical) of three polyphenolic compounds (namely esculetol, esculin and rutin whose chemical formulae are given in Table 1) in a weak acidic medium containing $10^{-3} \mathrm{M} \mathrm{NaCl}$ at $\mathrm{pH} 4$. Rutin, also called rutoside or quercetin-3-O-rutinoside, is a diglycoside of quercetin. This flavonoid is found in many fruits (especially the citrus fruits orange, grapefruit, lemon, and lime) or apple, peaches, and cereal as buckwheat seeds [18,19]. Esculin and esculetol belong to the family of coumarin. Particularly, esculin is a coumarin glucoside that naturally occurs in some trees or seeds (horse chestnut for example). Esculetol is the same molecule as esculin without the glucoside group [20]. These three molecules present three different

\footnotetext{
* Corresponding author

E-mail address: delphine.veys-renaux@univ-lorraine.fr (D. Veys-Renaux).
} 
Table 1

Chemical formulae of polyphenolic molecules tested as corrosion inhibitors.

Esculetol

configurations of the catechol group.

The electrochemical behaviour of steel in presence of these molecules was evaluated by using stationary electrochemical methods (corrosion potential measurements and polarization curves) and correlated to the characterisation of steel surfaces after immersion. The morphology of the surface layers was examined by Scanning Electron Microscopy (SEM) and the composition of the surface products was identified by Raman spectroscopy. On the other hand, solid iron polyphenolate compounds were prepared in aqueous solution and analysed by Raman spectroscopy and Synchrotron X-ray Absorption Spectroscopy in order to understand the coordination chemistry between the iron cation and the polyphenolic molecules.

\section{Experimental}

\subsection{Materials}

Low carbon steel plates (C: $0.32-0.39$ wt.\%, Mn: 0.5-0.8 wt.\%) used for further electrochemical characterization as well as surface analyses were first abraded on SiC paper (until particles size of $15 \mu \mathrm{m}$ ) and polished with a colloidal silica suspension until obtaining a mirror-like aspect.

The polyphenolic molecules tested as corrosion inhibitors are two coumarins: esculetol (6,7-dihydroxycoumarin 98\%, provided by Sigma Aldrich), esculin (esculin hydrate $87 \%$, provided by Acros Organics) and one flavonoid: rutin (rutin 97\%, provided by Acros Organics). Their chemical formulae are given in Table 1.

\subsection{Electrochemical measurements}

Due to the low water solubility of the tested compounds, the electrochemical characterisations were carried out in a water/ethanol (50/ 50) based medium containing $\mathrm{NaCl} 10^{-3} \mathrm{M}$. The inhibiting molecules were then added separately with a concentration of $10^{-3} \mathrm{M}$. The $\mathrm{pH}$ of all the solutions was adjusted to 4 by adding a small amount of $\mathrm{HCl}$. The corrosive solutions were aerated by bubbling industrial air. The circular working electrode surface $\left(3 \mathrm{~cm}^{2}\right)$ was horizontal, facing to a platinum grid used as counter electrode (mounted at a fixed distance of the reference an working electrodes). The reference electrode was a KCl-saturated calomel electrode (SCE). Electrochemical tests were performed at room temperature using a Versastat4 Potentiostat driven by Versastudio software.

The following experimental sequence was used:

- measurement of the corrosion potential $\left(E_{\text {corr }}\right)$ during $5 \mathrm{~h}$ in the corrosive medium, containing or not an inhibiting molecule,

- measurement of the anodic and cathodic polarization curves independently after $5 \mathrm{~h}$ immersion in the corrosive medium with a scan rate of $1 \mathrm{mV} \mathrm{s}^{-1}$.

\subsection{Synthesis of Fe-polyphenolate compounds}

\subsubsection{Treated steel coupons (formation of conversion layers)}

To investigate the surface products formed in the presence of polyphenolic molecules, steel pieces $(1 \mathrm{~cm} \times 1 \mathrm{~cm})$ were immersed during 5 days in a water/ethanol (50/50) medium containing separately the inhibiting molecules with a concentration of $10^{-3} \mathrm{M}$ and with a pH adjusted to 4 with $\mathrm{HCl}$. Thes immersions result in the formation of black conversion layers.

\subsubsection{Preparation of reference Fe-polyphenolate powders}

In order to study the complexation of iron with the polyphenolic molecules and the possible formation of insoluble precipitates on surface, Fe-polyphenolate compounds were synthesized using an iron chloride salt (anhydrous $\mathrm{FeCl}_{3}$, provided by Acros Organics) and the polyphenolic model molecules (rutin, esculetol, esculin) described above (Table 1). Iron chloride $10^{-2} \mathrm{M}$ and rutin or esculin or esculetol $10^{-2} \mathrm{M}$ were dissolved in water thanks to a moderate heating at $40^{\circ} \mathrm{C}$. The measured $\mathrm{pH}$ was around 2 . The $\mathrm{pH}$ was slowly increased by addition of $\mathrm{NaOH}$ solution $(0.1 \mathrm{M})$ until the precipitation of a black compound (occurring at $\mathrm{pH}=4$ ). After 1 week decantation at room temperature and fixed $\mathrm{pH}$ followed by $20 \mathrm{~min}$ of centrifugation at $4000 \mathrm{rpm}$, the resulting products were filtered and dried at $40{ }^{\circ} \mathrm{C}$ during 3 days. 3 black powders were obtained depending on the initial polyphenolic molecules. These precipitation reference compounds will be named Fe-esculin, Fe-rutin and Fe-esculetol.

\subsection{Morphological and structural characterization of Fe-polyphenolate compounds}

The treated surface and the synthesized reference powders were analysed by a combination of different analytical techniques described hereafter and including Scanning Electron Microscopy (SEM), Raman spectroscopy and X-Ray Diffraction (XRD).

SEM observations revealed the resulting morphologies of the treated surface, using a FEG-SEM Hitachi S4800, with an incident beam at a very low acceleration voltage $(1 \mathrm{kV})$ in order to be more sensitive to extreme surface.

XRD measurements were realized on the immersed pieces and powder compounds using a classical diffractometer (X'Pert Pro/ Philips), with a Cu Ka $\alpha_{1}$ source $\left(\lambda_{1}=1.54051 \AA\right)$.

Raman spectroscopy measurements were performed using a Labram Infinity spectrometer (Horiba Jobin Yvon) on both reference compounds powders and treated metallic coupons. The reference compounds powders (Fe-rutin, Fe-esculin and Fe-esculetol) slightly compacted on a glass slide were analysed using a $532 \mathrm{~nm}$ radiation from a frequency doubled Nd:YAG Laser with a laser power of about $100 \mu \mathrm{W}$ at the sample, and a spectral resolution of about $2 \mathrm{~cm}^{-1}$. The spectrometer is calibrated using the $520.5 \mathrm{~cm}^{-1}$ band of a Si crystal.

\subsection{Synchrotron X-ray absorption spectroscopy of the reference Fe- polyphenolate powders}

X-ray absorption spectroscopy measurements of reference Fe-polyphenolate compounds were realized on DiffAbs beamline at SOLEIL synchrotron (France). The main optical system of DiffAbs beamline, which gives a beam size about $300 \mu \mathrm{m} \times 300 \mu \mathrm{m}$ (FWHM) consists of a fixed-exit $\mathrm{Si}(111)$ double crystal monochromator and provides a 
focalization in the horizontal plane. Experiments were done in fluorescence mode at the iron K-edge over the whole XANES (X-ray Absorption Near Edge Structure) and EXAFS (Extended X-ray Absorption Fine Structure) domains. The intensity of the incident beam onto the sample was monitored using a thin Si photodiode, while the fluorescence signal was acquired using a Vortex 4E silicon drift detector, put at $90^{\circ}$ of the incident beam, i.e. $45^{\circ}$ of the sample surface. The energy scans at Fe K-edge were measured from 7050 to $7800 \mathrm{eV}$. The acquisition conditions were the following: steps of $1 \mathrm{eV}$ in the preedge region, $0.5 \mathrm{eV}$ in the edge domain and from 1 to $2 \mathrm{eV}$ in the EXAFS region. Counting time was set to $1 \mathrm{~s}$. The absolute energy of the spectra was referenced with a pure Fe metallic foil such that the first maximum of the derivative of the absorption spectrum was at $7112 \mathrm{eV}$.

Absorption spectra were processed using the Athena and Artemis software from Horae package (Iffefit program suite [21]). The pre-edge range background was removed using a linear baseline and the absorption background was removed using a cubic spline after the edge to obtain normalized spectra. The EXAFS signal (Chi spectra) was Fourier transformed from $\mathrm{k}$ to $\mathrm{R}$ space using a Hanning window. This operation results in a radial distribution function uncorrected from phase shift functions (displacement of about $0.4 \AA$ from crystallographic distances). The Fourier transform function was then fitted in order to determine the structural and chemical parameters. Multiple scattering effects were not taken into account.

\section{Results}

\subsection{Electrochemical behaviour}

The corrosion behaviour of steel in presence of the polyphenolic molecules was evaluated by stationary electrochemical methods: corrosion potential measurements as well as anodic and cathodic polarizations as reported respectively in Figs. 1 and 2.

The presence of rutin or esculin does not change the evolution of the corrosion potentials during the immersion of steel in the corrosive medium (Fig. 1). Firstly, the recorded values decrease and stabilize in the range of $-650 \mathrm{mV}$ vs SCE to $-600 \mathrm{mV}$ vs SCE after $1-2 \mathrm{~h}$. On the contrary, the presence of esculetol modifies the electrochemical interface on steel and increases the corrosion potential. Indeed, in the case of esculetol addition to the corrosive medium, the corrosion potential decrease stops after $30 \mathrm{~min}$ of immersion and the value stabilizes between $-450 \mathrm{mV}$ vs SCE and $-400 \mathrm{mV}$ vs SCE.

This potential increase due to the presence of esculetol in the corrosive medium is confirmed by the polarization curves drawn in Fig. 2 with a shift of the corrosion potential regarding the three tested other media. This difference can be correlated with the inhibition of the

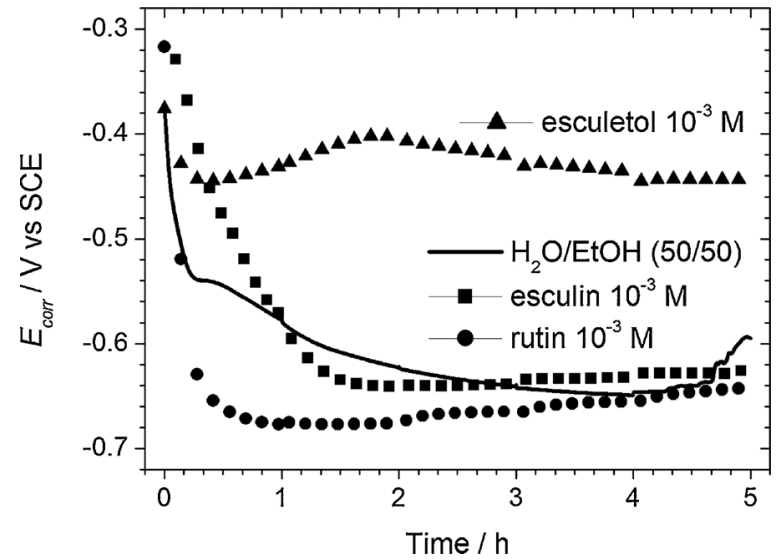

Fig. 1. Corrosion potential measurements during the immersion of steel in a water/ ethanol based electrolytic medium containing $\mathrm{NaCl}_{10}^{-3} \mathrm{M}$ and at $\mathrm{pH} 4$. Effect of the addition of polyphenolic model molecules: esculin, rutin, esculetol.

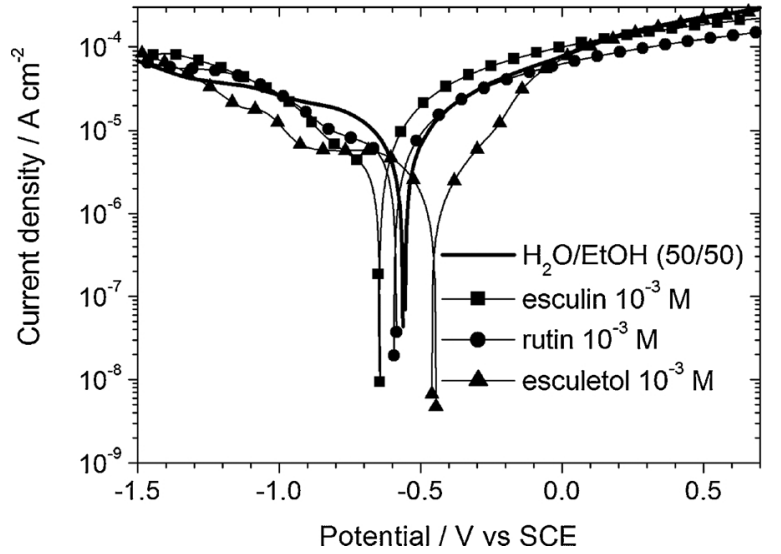

Fig. 2. Cathodic and anodic polarization curves recorded on steel after $5 \mathrm{~h}$ of immersion in a water/ethanol based electrolytic medium containing $\mathrm{NaCl}_{10} 0^{-3} \mathrm{M}$ and at $\mathrm{pH} 4$. Effect of the addition of polyphenolic model molecules: esculin, rutin, esculetol.

anodic current density relative to the reaction of iron oxidation observed in the electrolyte containing esculetol. In this specific case, the anodic current is reduced between $\mathrm{E}_{\text {corr }}$ and $\mathrm{E}_{\text {corr }}+200 \mathrm{mV}$, without the apparition of a real passivation plateau. Rutin and esculin have no effect on the anodic side of the polarization curve.

The cathodic side of the polarization curves corresponds to the oxygen reduction in this corrosive medium in which industrial air is bubbling. The addition of the three polyphenols induces a slight decrease of the cathodic current density which is divided by a factor 5 . This effect can be correlated with the antioxidant properties of the molecules, known as oxygen scavengers. Even if the cathodic current density is similar whatever the added polyphenol molecule, the cathodic range in which the effect of esculetol can be observed is larger (500 mV for esculetol against $200-300 \mathrm{mV}$ for rutin and esculin).

\subsection{Characterization of the conversion layers}

Without polyphenol molecules, the SEM observations carried out after immersion of steel in water/ethanol at pH 4 (Fig. 3a and b) show tangled needles and platelets homogeneously covering the surface, corresponding to the typical microscopic morphology of oxyhydroxide iron compounds as lepidocrocite, which is confirmed by XRD analysis (Fig. 4).

The addition of the three polyphenolic molecules modifies the surface morphology of the immersed steel (Fig. 3c-h) which becomes black, suggesting the formation of a conversion product. With the presence of esculin (Fig. 3c and d), a dusty, not homogeneous and not well-adherent deposit partially covers the surface. The XRD pattern of the formed product shows that this layer is partly constituted by iron oxyhydroxides, such as lepidocrocite and goethite.

In the cases of rutin (Fig. $3 \mathrm{e}$ and $\mathrm{f}$ ) and esculetol (Fig. $3 g$ and h), a coating consisting of two different stacked layers is observed (Fig. $3 \mathrm{f}$ and $\mathrm{h}$ ). It is amorphous or very poorly crystallized, as shown in the XRD patterns of Fig. 4. The internal part is made of needles with a finer morphology. The external part of the coating, strongly adherent to the intern one, is a compact and smooth layer with a thickness of about $300 \mathrm{~nm}$ in both cases. The main difference between the coatings grown on steel in presence of rutin or esculetol is their covering rate. Actually, even if the deposit formed in presence of esculetol appears as cracked in some places (Fig. 3h), it covers homogeneously the surface and is adherent (Fig. 3g). In the case of rutin (Fig. 3e), the coating grows away from the substrate and rolls up (Fig. 3f). Despite their amorphous (or poorly crystallized) character, these black products formed on the steel surface for all molecules are mainly constituted by iron, carbon and oxygen, according to qualitative EDX analysis. 


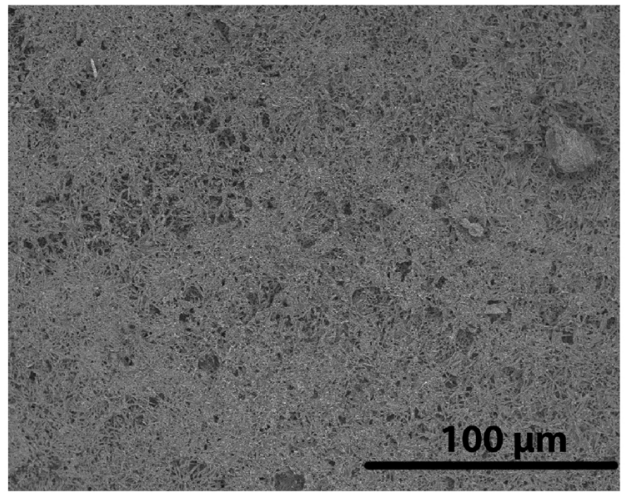

(a)

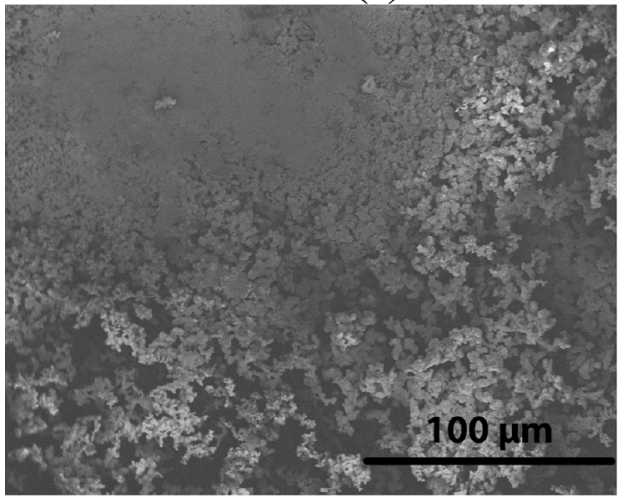

(c)

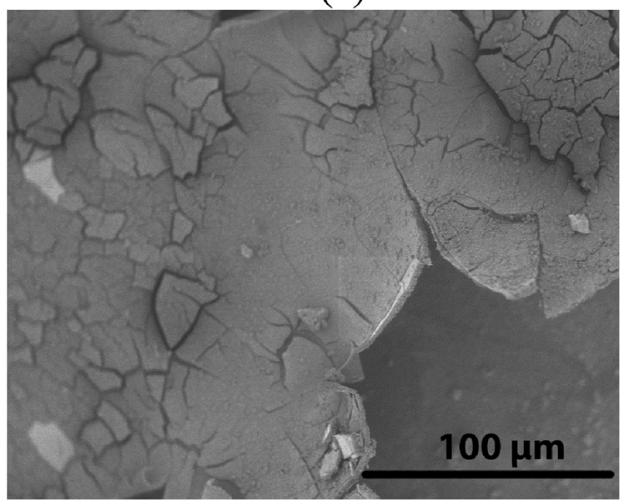

(e)

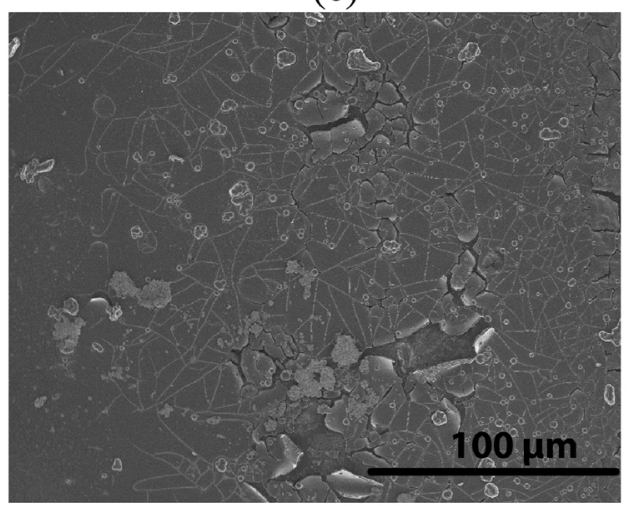

(g)

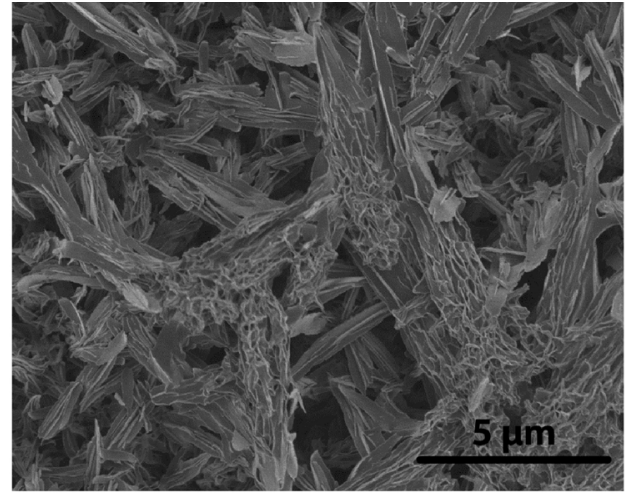

(b)

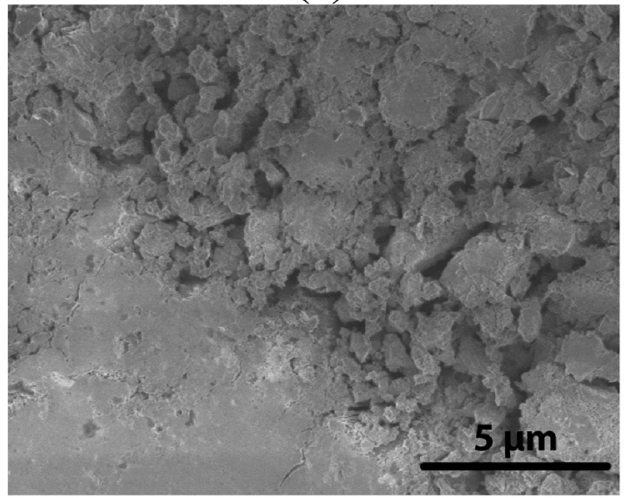

(d)

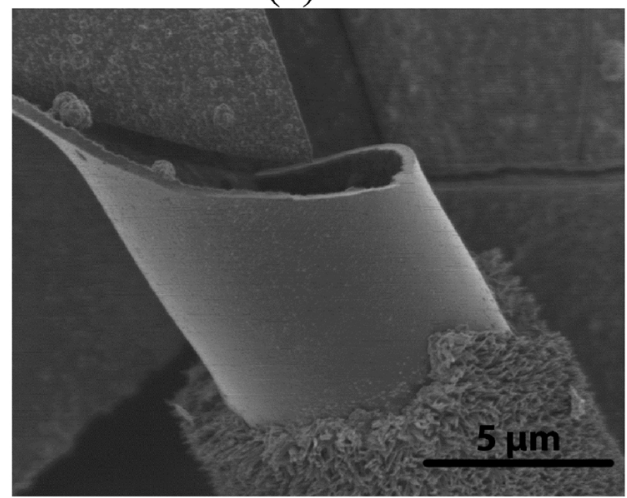

(f)

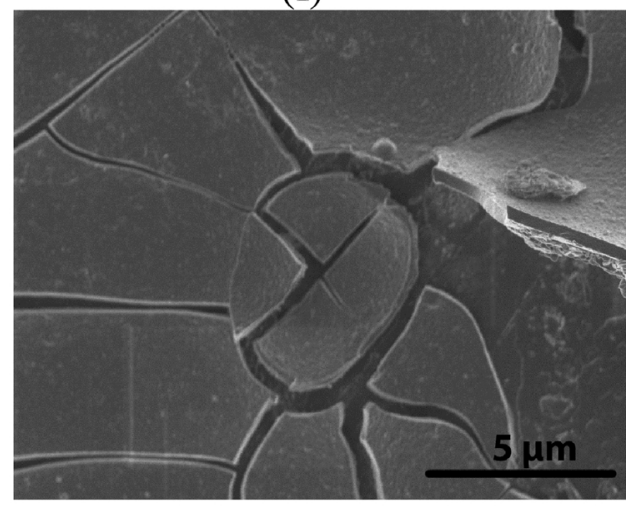

(h)

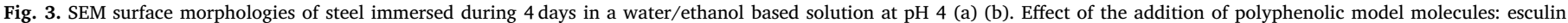
$10^{-3} \mathrm{M}$ (c) (d), rutin $10^{-3} \mathrm{M}(\mathrm{e})(\mathrm{f})$, esculetol $10^{-3} \mathrm{M}(\mathrm{g})(\mathrm{h})$. 


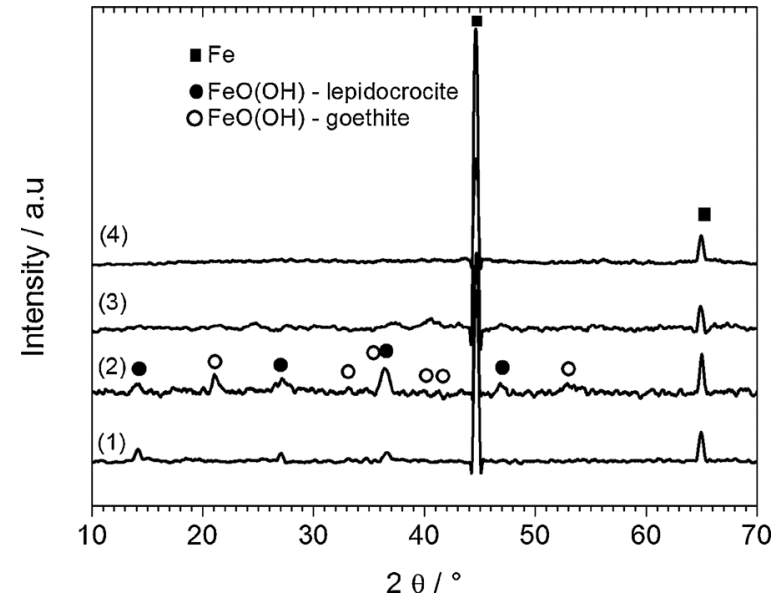

Fig. 4. X-Ray Diffraction patterns of steel immersed during 4 days in a water/ethanol based solution at $\mathrm{pH} 4$ (1). Effect of the addition of polyphenolic model molecules: esculin $10^{-3} \mathrm{M}(2)$, rutin $10^{-3} \mathrm{M}$ (3), esculetol $10^{-3} \mathrm{M}$ (4).

\subsection{Advanced structural analysis of conversion layers and reference Fe- polyphenolate powders}

Advanced spectroscopic analyses were performed in order to characterize the structure of the black conversion products precipitated on the surface, and especially the local chemical environment of iron cation in these products.

Measurements by Raman spectroscopy were realized on the surface of immersed steel and on the reference Fe-polyphenolate powders in order to determine if both are related to the same products.

Despite the very low amount of conversion product formed at the surface of the steel plates (thicknesses of about $300 \mathrm{~nm}$ ) meaningful Raman spectra were obtained. In Fig. 5 the Raman spectrum of each treated surface is compared to the relative reference powder (Fe-rutin, Fe-esculin, Fe-esculetol) and also to the initial compound used for synthesis.

This comparison shows similar Raman spectra on the reference powder products and on the immersed steel surfaces whatever the polyphenol molecule.

In contrary, the Raman spectra of pure molecules and solid ironpolyphenolate exhibit some significant differences. More precisely, the high-wavenumber Raman bands may be assigned to internal ligand polyphenol vibrations: at $1200-1600 \mathrm{~cm}^{-1}$ to $\mathrm{C}-\mathrm{C}$ or $\mathrm{C}=\mathrm{C}$ stretching, $\nu(\mathrm{C}-\mathrm{C}) / \nu(\mathrm{C}=\mathrm{C})$, and at $1000-1220 \mathrm{~cm}^{-1}$ to $\mathrm{C}-\mathrm{O}$ and $\mathrm{C}-\mathrm{H}$ deformation, as $\nu(\mathrm{CH})$ and $\nu(\mathrm{CO})[22,23]$. An assignment of the Raman bands of the pure molecules is detailed in the study of M. Paczkowska et al. for rutin [24]. In this high-wavenumber region, the most intense band for iron polyphenolate solid compounds is observed between 1450 and $1500 \mathrm{~cm}^{-1}$ [25-29] and may be characteristic for a catecholate ligand.

In the low-wavenumber region, the predominant bands between 550 and $650 \mathrm{~cm}^{-1}$ are characteristics of $\mathrm{Fe}-\mathrm{O}$ stretching vibrations with some contribution of the deformation of benzene ring associated to catecholate group. More precisely, the bands observed around $580 \mathrm{~cm}^{-1}$ and $600 \mathrm{~cm}^{-1}$ can be associated to stretching and deformation vibrations of the $\mathrm{Fe}(\mathrm{O}-\mathrm{C}-\mathrm{C}-\mathrm{O})$ chelate ring, as already demonstrated by the calculated vibration bands by L. Ohrstrom et al. [25].

Consequently, the Raman analysis proves the presence of iron polyphenolate conversion compounds on steel surface after immersion. In addition, the molecules are likely under a "catecholate-form", which suggests that the iron cation on the surface of steel could be mainly linked to the polyphenolic molecules by the catechol group.

In order to determine the local chemical environment around $\mathrm{Fe}$ atoms, analyses by X-ray absorption spectroscopy (XANES and EXAFS) at the Fe K-edge were performed. Taking into account the structural similarity between conversion layers and Fe-polyphenolate reference

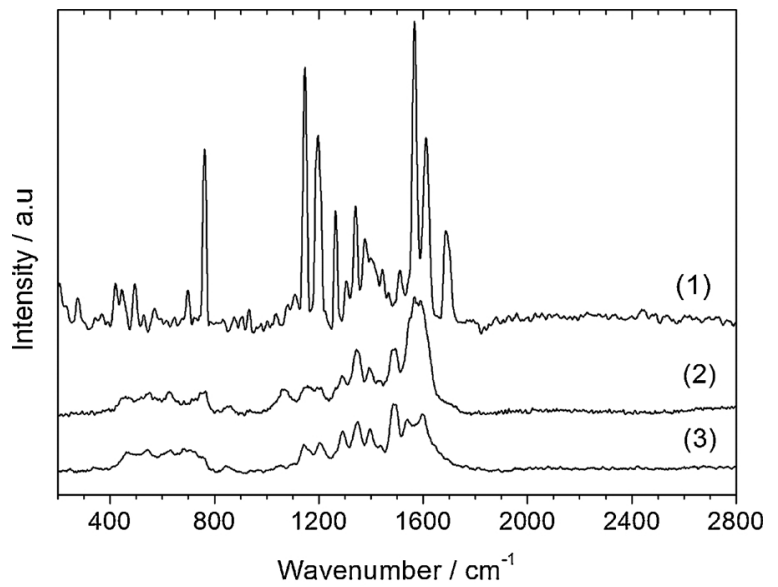

(a)

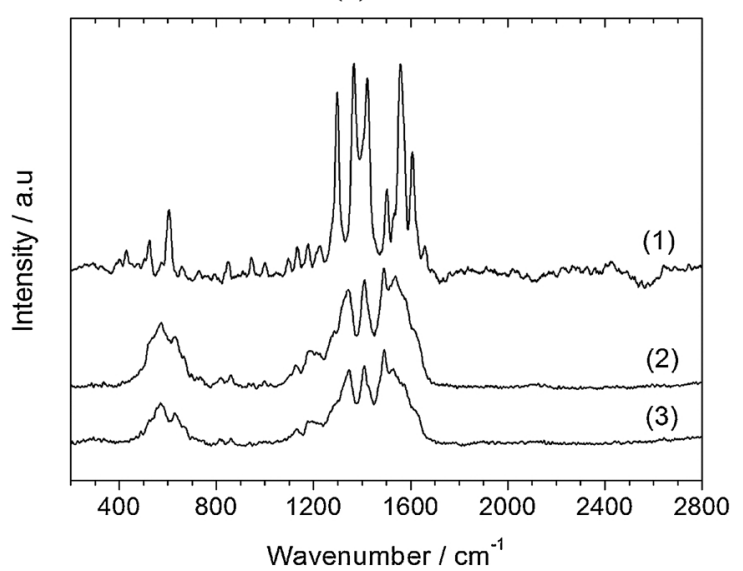

(b)

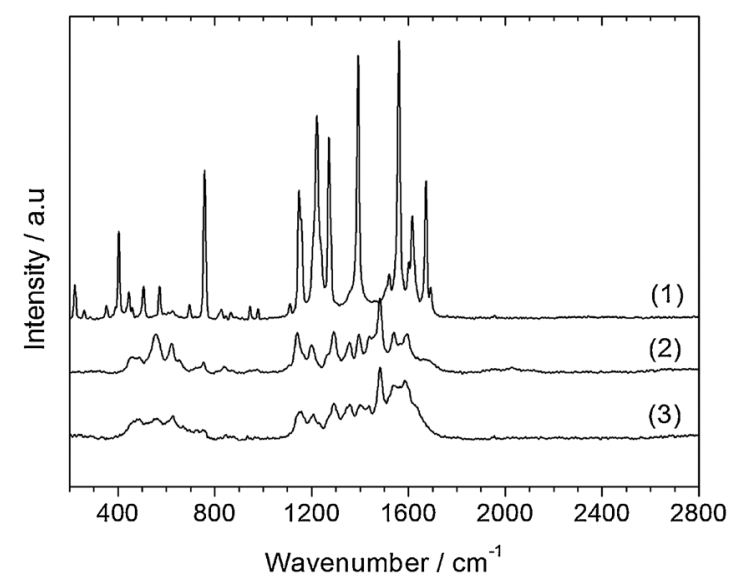

(c)

Fig. 5. Raman spectra obtained on the initial solid pure molecules (1), the solid synthesized compounds (2) and on the immersed surface (3) in the case of esculin (a), rutin (b), and esculetol (c) molecule.

compounds underlined by Raman spectroscopy, the XAS measurements were performed on the reference iron polyphenolate compounds (Feesculin, Fe-esculetol and Fe-rutine) which offer a sufficient amount of sample to achieve the analyses.

The XANES spectra are compared on Fig. 6 and the main features are described hereafter. The spectra exhibit quite the same features at the same position. The pre-edge is discretely visible at $7114 \mathrm{eV}$. The edge is characterized by a position given by the maximum of the derivative at $7126 \mathrm{eV}$ and a white line maximum at $7133 \mathrm{eV}$. After the edge, a shoulder-like feature is present at about $7147 \mathrm{eV}$. The amplitude 


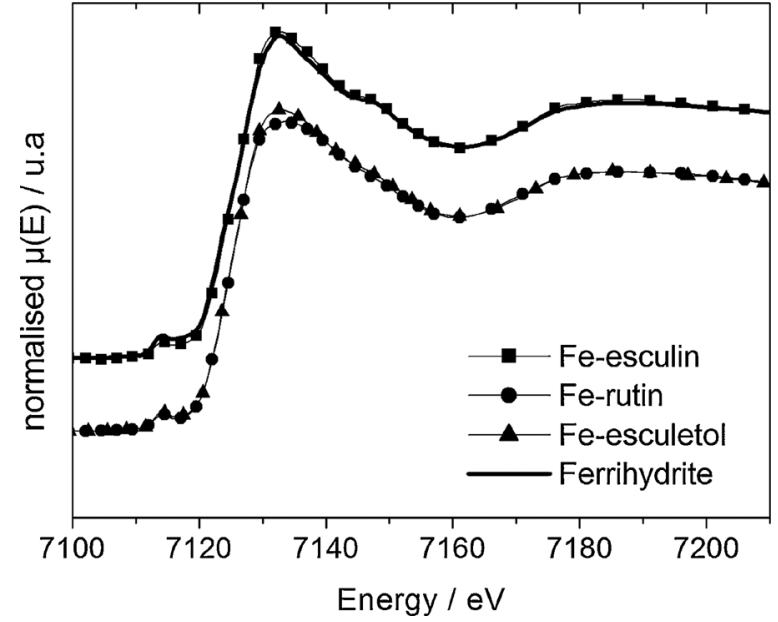

Fig. 6. XANES spectra measured at Fe K edge of the 3synthesized iron polyphenolates and comparison to the XANES spectra of ferrihydrite.

of this latter is greater in the case of Fe-esculin.

Both pre-edge and edge features reveal the electronic structure and coordination of iron atoms. The edge position corresponds to pure $\mathrm{Fe}^{3+}$ oxidation state compounds. As well-known for the first row transition elements, as $\mathrm{Fe}$, the white line is associated with the $1 \mathrm{~s}$ to $4 \mathrm{p}$ dipolar transition, and the shoulder following the white line corresponds to multiple scattering and/or multi-electronic excitations. The pre-edge features correspond to the $1 \mathrm{~s}$ level to $3 \mathrm{~d}$ state electronic transition that can be observed because of the orbital hybridization. The large oscillation at the beginning of the EXAFS region is mostly assigned to multiple scattering of the first neighbours, which is oxygen in the present case.

The extracted EXAFS spectra and the radial distribution function, obtained by Fourier transforming the EXAFS data, are reported on Figs. 7 and 8 respectively. The XANES and EXAFS spectra of the Feesculetol and Fe-rutin compounds (Fe-esculin,) are very close to each other, as shown on Figs. 6 and 7. The X-ray absorption signal of Feesculin compound is slightly different, and show a shoulder at $7150 \mathrm{eV}$ on the XANES spectra and at $5.5 \AA^{-1}$ on the EXAFS spectra, characteristic of the presence of an iron oxyhydroxide $\mathrm{FeOOH}$ (the example of ferrihydrite was chosen) [30,31].

Nevertheless, the chemical bonding environment of the iron cation in the three Fe-polyphenolate compounds can be well modelled with an octahedral coordination $\mathrm{FeO}_{6}$, as analysed by some authors [32,33], in particular by Z. Wang in the case of some polyphenols plant extracts

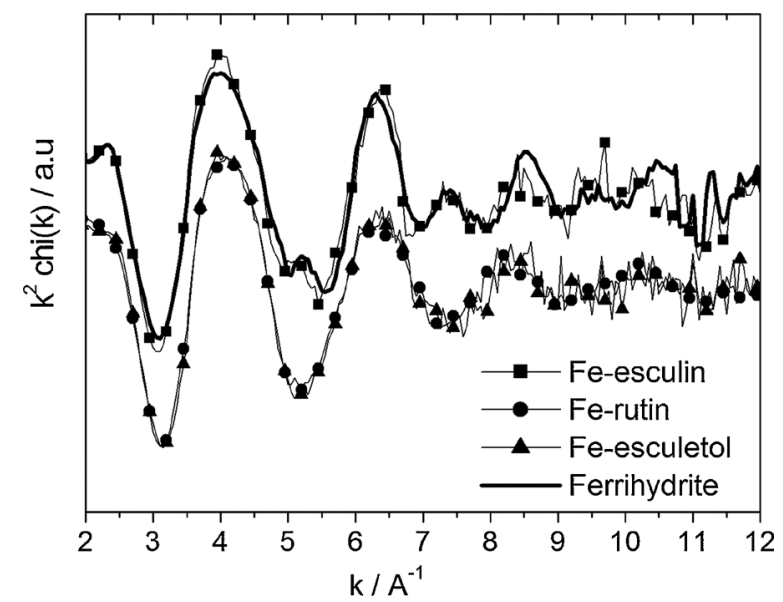

Fig. 7. EXAFS signals measured at Fe K edge of the 3synthesized iron polyphenolates and comparison to the EXAFS signal of ferrihydrite.

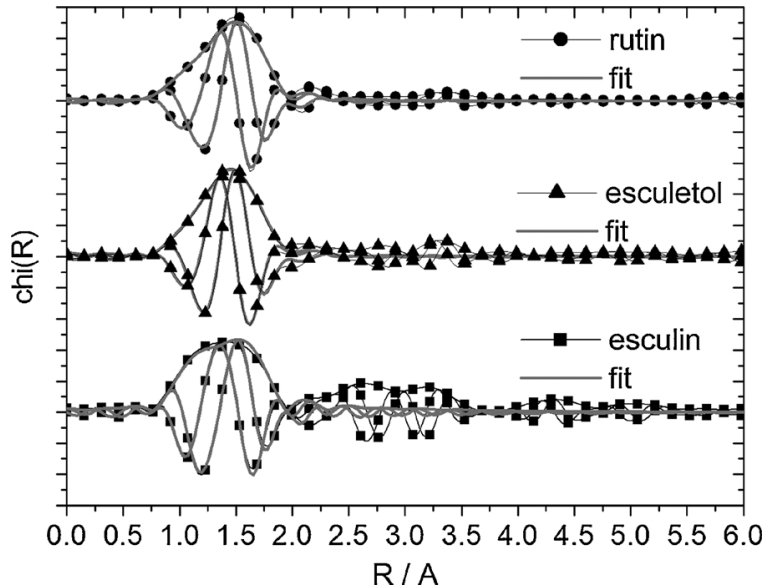

Fig. 8. FT signals measured at Fe K edge of the 3synthesized iron polyphenolates and best fits.

Table 2

Structural parameters derived from EXAFS at Fe K-edge.

\begin{tabular}{|c|c|c|c|c|c|c|c|}
\hline \multirow[t]{2}{*}{ elements } & \multirow[t]{2}{*}{$\mathrm{N}$} & \multicolumn{2}{|c|}{ esculin } & \multicolumn{2}{|l|}{ rutin } & \multicolumn{2}{|c|}{ esculetol } \\
\hline & & $\mathrm{R} / \AA ̊$ & ss & $\mathrm{R} / \AA ̊$ & ss & $\mathrm{R} / \AA ̊ \AA$ & ss \\
\hline O & 6 & 1.988 & 0.013 & 2.000 & 0.012 & 1.984 & 0.011 \\
\hline
\end{tabular}

[34].

With this octahedral coordination hypothesis, the EXAFS fit results of each compound are reported in Fig. 8 and Table 2. For the 3 compounds, the first peak of the Fourier Transform (FT), assigned to the ligand sphere of $\mathrm{O}$ atoms surrounding $\mathrm{Fe}$ atoms, reveals that the $\mathrm{Fe}^{3+}$ cation is the centre of an octahedron composed by 6 oxygen atoms at a distance of about $1.98 \AA$ to $2 \AA$. The next visible peaks, expected to relate mainly to the next coordination shell, can correspond to the $\mathrm{Fe}-\mathrm{Fe}$ bonds. The Fe-Fe distances can be evaluated between $3 \AA$ to $3.5 \AA$ for all compounds. These atomic distance values are coherent with many iron polyphenolate compounds, already studied by X-ray absorption spectroscopy [33].

\section{Discussion}

According to the results obtained in this study, steel surfaces can be chemically converted into iron polyphenolate black compounds in presence of polyphenolic molecules at a weak acidic $\mathrm{pH}(\mathrm{pH} 4)$. Actually, SEM observations reveal the formation of a $300 \mathrm{~nm}$ thick dense organometallic film after immersion of steel in solutions containing esculetol or rutin, without the formation of oxyhydroxyde. By contrast, the compounds formed on steel surface in presence of esculin are composed by both iron oxyhydroxide corrosion and iron polyphenolate compounds.

The analysis of Raman spectra confirmed that the surface products are formed by precipitation of ferric cations with polyphenolic molecules. The energy position of absorption Fe K-edge determined by XAS proves the pure $\mathrm{Fe}^{3+}$ oxidation state in the three Fe-polyphenolate products, and the chemical environment of $\mathrm{Fe}^{3+}$ can be well-described by an octahedron of oxygen atoms with a calculated distance $\mathrm{Fe}-\mathrm{O}$ in the range $1.98-2.00 \AA$ in the three cases.

In addition, Raman spectroscopy suggests that the iron coordination by oxygen atoms is probably made through the catechol group present on one of the benzene ring of esculetol, esculin and rutin. Actually it is known that catechol acts as a bidentate ligand and can form an octahedral tris-catecholate complex with iron [32]. In literature, theoretical calculations give Fe-O distances of $1.92 \AA$ [33], in good agreement with the XAS results of the present study. 


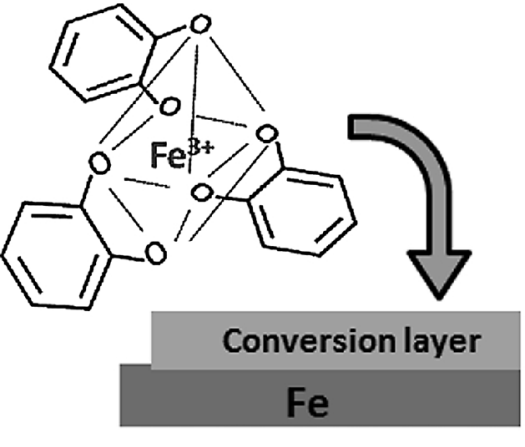

a)

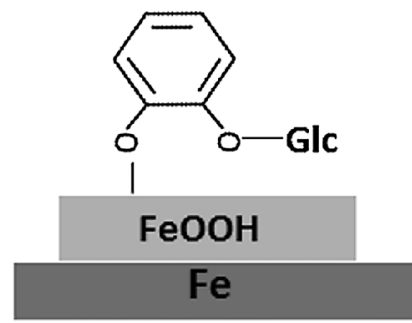

b)

Fig. 9. Schematic representation of corrosion inhibition mechanism on steel by polyphenol containing free catechol group (a) and containing glycosyled-catechol group (b).

Finally, rutin and esculetol induce at $\mathrm{pH} 4$ the precipitation of a thin layer of conversion organometallic products on iron surface in which $\mathrm{Fe}^{3+}$ is likely coordinated to the organic molecules through the catechol groups, as illustrated in Fig. 9a.

In the case of esculin, whereas the esculin molecule is present on the steel surface, as shown by the Raman spectroscopy analysis, the iron oxyhydroxide remains one of the main products formed at $\mathrm{pH} 4$. The precipitation of Fe-esculin phenolate compound is more difficult and the esculin molecule seems to be mainly adsorbed on the iron oxyhydroxide surface (Fig. 9b). Actually, the coordination of the ferric cation with catecholate group of esculin seems to be impeded because of the glucosyl substitution on the benzene ring.

From the electrochemical point of view, the kinetic of oxygen reduction is reduced in the three cases in the same proportion, namely by a factor 2-3. Indeed esculin, rutin and esculetol are antioxidant compounds known to have a radical scavenging activity, so that the free radicals produced as intermediate species during oxygen reduction can be entrapped. This antioxidant activity is related to the catechol oxidation into semiquinone and benzoquinone. However, the formation of complex with metallic species affects the antioxidant activity of organic molecules and can decrease the catechol group scavenging properties [34]. This could explain the relatively low corrosion inhibition efficiency observed in the three cases and the similar decrease of the cathodic current density whatever the involved molecules.

Moreover, the formation of a well-covering and adherent tridimensional film induces both a physical barrier to the diffusion of oxygen until the metallic surface, and an inhibition of the anodic process. The protection efficiency against corrosion brought by the film obviously depends on its thickness and adherence. In the case of esculetol, the compactness of the formed layer leads to both a cathodic and anodic inhibition. In the case of rutin molecule, the conversion film formed on iron is not flat and its adherence is poor. Therefore, although the formation of a tri dimensional film in this case, its efficiency remains weak.

\section{Conclusion}

In weak acidic conditions, iron polyphenolate conversion films can be formed on the surface of iron by precipitation of iron (III) cations with polyphenolic molecules containing a free catechol group (esculetol, rutin), which is a bidentate ligand complexing iron in a tris-catecholate octahedron. The structure of the polyphenolic organic molecule affects the morphological properties of the conversion film. Only esculetol enables the growth of a well covering and adherent film and therefore induces an anodic inhibition of the corrosion process.

When the catechol group is blocked by a glucoside substitution, the formation of conversion film with iron polyphenolate compounds is more difficult on the steel surface.

From a corrosion point of view, the free-radical scavenging properties of these molecules induce a decrease of the oxygen reduction kinetic, similar with film-forming molecules (rutin, esculetol) and a non-forming one (esculin).

\section{Acknowledgements}

The authors would like to thank the French "Ministère de la Culture et de la Communication" and "Agence Nationale de la Recherche" for funding this study in the PNRCC project entitled Carbotan. Lise Salsi from the "Service Commun de Microanalyses" located at the "Faculté des Sciences et Techniques" in Nancy is greatly acknowledged for SEM observations as well as the DiffAbs beamline team at "Soleil Synchrotron" for experimental support in XAS measurements.

\section{References}

[1] S. Quideau, D. Deffieux, C. Douat-Casassus, L. Pouységu, Plant polyphenols: chemical properties, biological activities, and synthesis, Angew. Chem. Int. Ed. 50 (3) (2011) 586-621.

[2] L. Pourcel, J.M. Routaboul, V. Cheynier, L. Lepiniec, I. Debeaujon, Flavonoid oxidation in plants: from biochemical properties to physiological functions, Trends Plant Sci. 12 (1) (2007) 29-36.

[3] N. Schweigert, A.J.B. Zehnder, R.I.L. Eggen, Chemical properties of catechols and their molecular modes of toxic action in cells, from microorganisms to mammals, Environ. Microbiol. 3 (2) (2001) 81-91.

[4] L.K. Partridge, A.C. Tansley, A.S. Porter, The adsorption of coumarin at solid metal electrodes, Electrochim. Acta 12 (12) (1967) 1573-1578.

[5] Y.M. Chiou, L. Que Jr, Structure of a mononuclear iron(II)-Catecholate complex and its relevance to the extradiol-cleaving catechol dioxygenases, Inorg. Chem. 34 (14) (1995) 3577-3578.

[6] F.E. Aragão Catunda Jr., M. Francisco de Araujo, A.M. Granero, F.J. Arévalo, M. Geraldo de Carvalho, M.A. Zón, H. Fernández, The redox thermodynamics and kinetics of flavonoid rutin adsorbed at glassy carbon electrodes by stripping square wave voltammetry, Electrochim. Acta 56 (27) (2011) 9707-9713.

[7] M.E. Cass, T.M. Garrett, K.N. Raymond, The salicylate mode of bonding in protonated ferric enterobactin analogs, J. Am. Chem. Soc. 111 (1989) 1677-1682.

[8] A.M. Albrecht-Gary, A.L. Crumbliss, Coordination chemistry of siderophores: thermodynamics and kinetics of iron chelation and release, Met. Ions Biol. Syst. 35 (1998) 239-327.

[9] A.M. Abdel-Gaber, B.A. Abd-El-Nabey, I.M. Sidahmed, A.M. El-Zayady, M. Saadawy, Inhibitive action of some plant extracts on the corrosion of steel in acidic media, Corros. Sci. 48 (2006) 2765-2779.

[10] E.E. Oguzie, Evaluation of the inhibitive effect of some plant extracts on the acid corrosion of mild steel, Corros. Sci. 50 (2008) 2993-2998.

[11] M. Lebrini, F. Robert, A. Lecante, C. Roos, Corrosion inhibition of C38 steel in $1 \mathrm{M}$ hydrochloric acid medium by alkaloids extract from Oxandra asbeckii plant, Corros. Sci. 53 (2011) 687-695.

[12] S. Deng, X. Li, Inhibition by Ginkgo leaves extract of the corrosion of steel in $\mathrm{HCl}$ and $\mathrm{H}_{2} \mathrm{SO}_{4}$ solutions, Corros. Sci. 55 (2012) 407-415.

[13] J.C. da Rocha, J.C. da Cunha Ponciano Gomes, E. D'Elia, Corrosion inhibition of carbon steel in hydrochloric acid solution by fruit peel aqueous extracts, Corros. Sci. 52 (2010) 234-2348.

[14] M. Behpoura, S.M. Ghoreishia, M. Khayatkashania, N. Soltanib, Green approach to corrosion inhibition of mild steel in two acidic solutions by the extract of Punica granatum peel and main constituents, Mater. Chem. Phys. 131 (2012) 621-633.

[15] A.A. Rahim, E. Rocca, J. Steinmetz, M.J. Kassim, R. Adnan, M. Sani Ibrahim, Mangrove tannins and their flavonoid monomers as alternative steel corrosion inhibitors in acidic medium, Corros. Sci. 49 (2007) 402-417.

[16] N. M’hiri, D. Veys-Renaux, E. Rocca, I. Ioannou, N. Mihoubi Boudhrioua, M. Ghoul, 
Corrosion inhibition of carbon steel in acidic medium by orange peel extract and its main antioxidant compounds, Corros. Sci. 102 (2016) 55-62.

[17] F. Suedile, F. Robert, C. Roos, M. Lebrini, Corrosion inhibition of zinc by Mansoa alliacea plant extract in sodium chloride media: extraction, Characterization and Electrochemical, Electrochim. Acta 133 (2014) 631-638.

[18] S. Kreft, M. Knapp, I. Kreft, Extraction of rutin from buckwheat (Fagopyrum esculentum Moench) seeds and determination by capillary electrophoresis, J. Agric. Food Chem. 47 (11) (1999) 4649-4652.

[19] S. Chang, C. Tan, E.N. Frankel, D.M. Barrett, Low-density lipoprotein antioxidant activity of phenolic compounds and polyphenol oxidase activity in selected clingstone peach cultivars, J. Agric. Food Chem. 48 (2) (2000) 147-151.

[20] B. Buszewski, S. Kawkab, Z. Suprynowicza, T. Wolskib, Simultaneous isolation of Rutin and Esculin from plant material and drugs using solid-phase extraction, J. Pharm. Biomed. 11 (3) (1993) 211-215.

[21] M. Newville, IFEFFIT: interactive XAFS analysis and FEFF fitting, J. Synchrotron Radiat. 8 (2001) 322-324.

[22] F. Hartl, P. Barbaro, I.M. Bell, R.J.H. Clark, T.L. Snoeck, A. Vlcek Jr., Valence localization in $[\mathrm{M}($ triphos)(3,5-di-tert-butyl-catecholate) $]+$ ions $(\mathrm{M}=\mathrm{Co}$, Rh or Ir) probed by resonance Raman spectroscopy, Inorg. Chim. Acta 252 (1996) 157-166.

[23] T.B. Karpishin, M.S. Gebhard, E.I. Solomon, K.N. Raymond, Spectroscopic studies of the electronic structure of iron(III) tris(catecholates), J. Am. Chem. Soc. 113 (8) (1991) 2977-2984.

[24] M. Paczkowska, K. Lewandowska, W. Bednarski, M. Mizera, A. Podborska, A. Krause, J. Cielecka-Piontek, Application of spectroscopic methods for identification (FT-IR, Raman spectroscopy) and determination (UV, EPR) of quercetin-3-Orutinoside, Experimental and DFT based approach, Spectrochim. Acta A 140 (2015) 132-139.

[25] L. Ohrstrom, I. Michaud-Soret, Quantum chemical approach to the assignment of iron-catecholate vibrations and isotopic substitution shifts, J. Am. Chem. Soc. 118 (1996) 3283-3284.
[26] J. Ling, M. Sahlin, B.M. Sjoberg, T.M. Loehr, J. Sanders-Loehr, Dioxygen is the source of the p-Oxo bridge in iron ribonucleotide, reductase, J. Biol. Chem. 269 (8) (1994) 5595-5601.

[27] W.J. Barreto, R.A. Ando, P.S. Santos, W.P. Silva, I.R. Preparation, UV-vis EPR and resonance raman study of $\mathrm{Fe}, \mathrm{Ni} \mathrm{Co}$ and $\mathrm{Zn}$ dioxolene complexes, Spectrochim. Acta A 68 (2007) 612-618.

[28] S. Salama, J.D. Stong, J.B. Neilands, T.G. Spiro, Electronic and resonance Raman spectra of iron(III) complexes of enterobactin, catechol, and N-methyl-2, 3-dihydroxybenzamide, Biochemistry 17 (18) (1978) 3781-3785.

[29] I. Michaud-Soret, K.K. Andersson, L. Que Jr., Resonance raman studies of catecholate and phenolate complexes of recombinant human tyrosine hydroxylase, Biochemistry 34 (16) (1995) 5504-5510.

[30] J. Monnier, S. Reguer, E. Foy, D. Testemale, F. Mirambet, M. Saheb, P. Dillmann, I. Guillot, XAS and XRD in situ characterisation of reduction and reoxidation processes of iron corrosion products involved in atmospheric corrosion, Corros. Sci. 78 (2014) 293-303.

[31] F. Mirambet, S. Reguer, E. Rocca, S. Hollner, D. Testemale, A complementary set of electrochemical and X-ray synchrotron techniques to determine the passivation mechanism of iron treated in a new corrosion inhibitor solution specifically developed for the preservation of metallic artefacts, Appl. Phys. A-Mater. 99 (2) (2010) 341-349.

[32] A. Avdeef, S.R. Sofen, T.L. Bregante, K.N. Raymond, Coordination chemistry of microbial iron transport compounds. 9. Stability constants for catechol models of enterobactin, J. Am. Chem. Soc. 100 (1978) 5362-5370.

[33] I. Kara, Y. Kara, A.O. Kiraz, R. Mammadov, Theoretical calculations of a compound formed by $\mathrm{Fe}^{3+}$ and tris(catechol), Spectrochim. Acta A 149 (2015) 592-599.

[34] J.M. Dimitric Markovic, Z.S. Markovic, T.B. Brdaric, V.M. Pavelkic, M.B. Jadranin, Iron complexes of dietary flavonoids: combined spectroscopic and mechanistic study of their free radical scavenging activity, Food Chem. 129 (2011) 1567-1577. 